\title{
Are zinc transporter type 8 antibodies a marker of autoimmune thyroiditis in non-obese adults with new-onset diabetes?
}

\section{Anita Rogowicz-Frontczak', Dorota Zozulińska-Ziółkiewicz', Monika Litwinowicz², Paweł Niedźwiecki ${ }^{1}$, Krystyna Wyka ${ }^{3}$ and Bogna Wierusz-Wysocka ${ }^{1}$}

Departments of ${ }^{1}$ Internal Medicine and Diabetology and ${ }^{2}$ Internal Diseases, Metabolic Disorders and Dietetics, Poznan University of Medical Sciences, Mickiewicza 2, 60-834 Poznan, Poland and ${ }^{3}$ Medical University of Lodz, Lodz, Poland
Correspondence

should be addressed to

A Rogowicz-Frontczak

Email

anitrog@gmail.com

\begin{abstract}
Objective: The diagnosis of autoimmune diabetes in non-obese adults is based on the detection of glutamic acid decarboxylase autoantibodies (GADA), islet cell antibodies (ICA) and antibodies to tyrosine phosphatase (IA-2A). Zinc transporter 8 ( $\mathrm{ZnT} 8$ ) has been identified as a new autoantigen in patients with type 1 diabetes mellitus. The coincidence of autoimmune thyroiditis (AITD) with diabetes is common; therefore, screening of TSH and thyroid peroxidase antibodies (ATPO) is recommended during the diagnosis of diabetes. In this study, we determined whether the occurrence of islet autoantibodies is associated with a positive titre of ATPO in newly diagnosed adult-onset autoimmune diabetic patients. Design and methods: The study involved 80 non-obese adults aged 44 (interquartile range (IQR): 37-51) years with a BMI of 24.0 (IQR: $22.2-26.0$ ) kg/m² and new-onset diabetes. The markers of autoimmune diabetes (GADA, ICA, IA-2A and ZnT8A), TSH and thyroid peroxidase antibodies (ATPO) were evaluated.

Results: In the study population, $70 \%(n=56)$ of the subjects were positive for at least one of the four assessed markers of autoimmune diabetes (83.9\% GADA, 62.5\% ICA, 42.8\% IA-2A and 33\% ZnT8A) and 37.5\% of the subjects were positive for ATPO. The ZnT8A-positive subjects had higher ATPO titres than the ZnT8A-negative subjects (172.7 (IQR: 0.36-410.4) vs 92.4 (IQR: $0-23.7$ ) IU/ml, $P=0.001$ ). Based on the assessed islet autoantibodies, the occurrence of positive ZnT8A and GADA was found to be related to a positive titre of ATPO using logistic regression (OR=5.48, 95\% Cl: $1.65-18.14, P=0.006$ and $\mathrm{OR}=3.42,95 \% \mathrm{Cl}: 1.09-10.71, P=0.03$ respectively).

Conclusions: In non-obese adults with new-onset diabetes, the presence of GADA and especially ZnT8 autoantibodies increases the risk of AITD.
\end{abstract}

\section{Introduction}

Autoimmune diabetes is a common form of diabetes in childhood and adolescence, but it could appear at any age (1). In adults, usually the failure of $\beta$-cells tends to be slower and the onset of the disease occurs frequently without ketoacidosis. For this reason, some of these patients could be inappropriately classified as having type 2 diabetes mellitus (T2DM) (2). Therefore, the latent autoimmune diabetes in adults (LADA) was defined as diabetes diagnosed in patients aged $>35$ years, identified by the presence of islet autoantibodies $(3,4)$. During the natural development of the disease, most of the cases of LADA progress to insulin dependency $(5,6)$. The possibility of LADA should be mainly considered among patients without obesity and other features of the metabolic syndrome or with the coincidence of other autoimmunological diseases. LADA individuals are characterised by lower fasting and stimulated C-peptide levels compared with individuals with classic T2DM (7). (c) 2014 European Society of Endocrinology Printed in Great Britain
Published by Bioscientifica Ltd. 
The final diagnostic criterion for LADA comprises the detection of autoantibodies. Glutamic acid decarboxylase antibodies (GADA) and islet cell antibodies (ICA) are described to be the most common antibodies present in LADA individuals $(8,9,10)$. Studies carried out previously have also revealed the occurrence of other antibodies among LADA patients such as antibodies to tyrosine phosphatase (IA-2A) and autoantibodies to insulin (IAA) $(8,10,11)$. Recently, zinc transporter 8 (ZnT8) has been identified as a novel autoantigen contributing to the diagnostic sensitivity of autoimmune diabetes. The gene responsible for coding ZnT8 is named SCL3OA8 and is localised on chromosome 8q24.11 (12). ZnT8 antibodies (ZnT8A) are present in both juvenile-onset and adultonset autoimmune diabetic patients $(9,13)$.

In LADA patients, autoimmune thyroiditis (AITD) is the most frequent concomitant autoimmune disease $(14,15)$. Therefore, routine screening for anti-thyroid autoimmunity is recommended during the diagnosis of diabetes. The screening performed should at least include thyroid-stimulating hormone (TSH) and anti-thyroid peroxidase autoantibody (ATPO) evaluation (16). In light of the previous studies, especially individuals with high GADA titres should be assessed to determine possible thyroid dysfunction (17).

The aim of this study was to evaluate the classic islet autoantibodies (GADA, ICA and IA-2A) and a new marker of autoimmunity in diabetes (ZnT8A) in non-obese newly diagnosed diabetic patients aged $>35$ years to determine their association with ATPO.

\section{Subjects and methods}

We enrolled consecutive, non-obese individuals aged $>35$ years with newly diagnosed diabetes, without previous therapy hospitalised in the Department of Internal Medicine and Diabetology, Poznan University of Medical Sciences, in the years 2009-2012.

Inclusion criteria were as follows: recognition of diabetes based on the classification of National Diabetes Data Group and World Health Organisation (1), Caucasian race, and aged 35-65 years.

Exclusion criteria were as follows: BMI $\geq 30 \mathrm{~kg} / \mathrm{m}^{2}$, cancer, hepatic failure (aminotransferase levels two times above the normal range) or diagnosed viral hepatitis $B$ (HBV) and viral hepatitis C (HCV), renal failure (estimated glomerular filtration rate (eGFR) $<60 \mathrm{ml} / \mathrm{min}$ per $1.72 \mathrm{~m}^{2}$ ), chronic pancreatitis, anaemia (haemoglobin level $<6.8 \mathrm{mmol} / \mathrm{l}$ ) and use of drugs affecting glucose metabolism and history of alcohol abuse (defined as consumption of more than $20 \mathrm{~g}$ of ethanol per day for a woman and more than $40 \mathrm{~g}$ for a man).

All the subjects were informed about the aim of the study and written informed consent was obtained from them. The study was approved by the Ethical Committee of Poznan University of Medical Sciences (793/09). All applicable institutional and governmental regulations concerning the ethical use of human volunteers were followed during the study.

After fulfilment of the exclusion criteria, a total of 80 patients (31 women and 49 men), with median age of onset of 44 years (interquartile range (IQR): 37-51) and median BMI of 24.0 (IQR: 22.2-26.0) $\mathrm{kg} / \mathrm{m}^{2}$, were recruited. There was no clinical manifestation of other autoimmune diseases besides thyroiditis in the study population. We investigated for the presence of ICA, GADA, IA-2A and ZnT8A. Furthermore, TSH and thyroid peroxidase antibodies (ATPO) were also evaluated.

According to the occurrence of the analysed islet autoantibodies, we divided the subjects into a group with at least one of the assessed autoantibodies (LADA) and a group without autoantibodies. Furthermore, for better analysis of the usefulness of ZnT8A, we divided LADA patients into a ZnT8A-positive group and a ZnT8Anegative group. Eventually, all the enrolled patients were treated with insulin, with some of them being treated additionally with metformin. The clinical characteristics of the study subjects are summarised in Table 1.

Anthropometric data included weight, height and waist circumference and were obtained using standardised techniques. Blood pressure was recorded in the right arm, with the subject in a sitting position, using a mercury sphygmomanometer. Two readings were taken 5 min apart, and the mean of the two readings was taken as the blood pressure.

Blood samples were collected in a fasting state defined as no caloric intake for at least $8 \mathrm{~h}$. Serum concentrations of glucose, HDL cholesterol, LDL cholesterol, triglycerides and creatinine were measured using standard methods. The concentration of HbA1c was measured using HPLC. The eGFR was calculated using the Modification of Diet in Renal Disease (MDRD) study equation.

In all the examined patients, we determined serum fasting and stimulating (6 min after i.v. injection of $1 \mathrm{mg}$ glucagon) C-peptide concentrations. Serum C-peptide concentrations were determined using an immunoenzymatic method (IMMULITE, DPC, Erlangen, Germany).

\section{Islet autoantibody assays}

ICA were detected using indirect immunofluorescence tests carried out on cryostat sections of the pancreas of a donor 
Table 1 Clinical characteristics of the study population and comparison between the group with at least one of the assessed autoantibodies and the group without autoantibodies (median and IQR or mean \pm s.D. or percentage of patients)

\begin{tabular}{|c|c|c|c|c|}
\hline & All patients & $\begin{array}{l}\text { Islet autoantibody- } \\
\text { negative group }\end{array}$ & $\begin{array}{l}\text { Group with one or more } \\
\text { of the assessed islet } \\
\text { autoantibodies }\end{array}$ & $P$ value* \\
\hline$n$ & 80 & 24 & 56 & - \\
\hline Sex (women/men) & $31 / 49$ & $8 / 16$ & $23 / 33$ & 0.51 \\
\hline Age (years) & $44(37-51)$ & $46(40-52)$ & $42(37-51)$ & 0.24 \\
\hline BMI $\left(\mathrm{kg} / \mathrm{m}^{2}\right)$ & $24.0(22.2-26.0)$ & $24.8(22.5-26.0)$ & $24.0(22.2-26.0)$ & 0.26 \\
\hline Waist $(\mathrm{cm})$, women & $80.5 \pm 10.0$ & $84.8 \pm 11.9$ & $79.1 \pm 9.2$ & 0.16 \\
\hline Waist (cm), men & $92.5 \pm 8.8$ & $95.9 \pm 8.3$ & $90.9 \pm 8.8$ & 0.08 \\
\hline $\mathrm{SBP}(\mathrm{mmHg})$ & $115(110-120)$ & $120(110-130)$ & $110(110-120)$ & 0.11 \\
\hline $\mathrm{DBP}(\mathrm{mmHg})$ & $70(70-80)$ & $80(70-80)$ & $70(70-80)$ & 0.23 \\
\hline $\mathrm{HbA1c}(\%)$ & $11.1 \pm 2.5$ & $9.9 \pm 2.5$ & $11.4 \pm 2.4$ & 0.01 \\
\hline Cholesterol $(\mathrm{mmol} / \mathrm{l})$ & $5.3 \pm 1.3$ & $5.8 \pm 1.7$ & $5.0 \pm 1.0$ & 0.03 \\
\hline TG $(\mathrm{mmol} / \mathrm{l})$ & $1.8(0.9-1.6)$ & $1.4(1.0-1.7)$ & $1.1(0.9-1.5)$ & 0.46 \\
\hline LDL (mmol/l) & $3.3 \pm 0.9$ & $3.2 \pm 0.9$ & $3.3 \pm 0.9$ & 0.78 \\
\hline HDL (mmol/l) & $1.3 \pm 0.4$ & $1.4 \pm 0.4$ & $1.3 \pm 0.5$ & 0.30 \\
\hline Creatinine (mg/dl) & $0.80 \pm 0.14$ & $0.77 \pm 0.12$ & $0.8 \pm 0.14$ & 0.37 \\
\hline GFR (MDRD) (ml/min) & $103.9 \pm 24.2$ & $108.5 \pm 22.4$ & $101.9 \pm 24.9$ & 0.27 \\
\hline Fasting C-peptide (ng/ml) & $1.3 \pm 0.8$ & $1.7 \pm 0.9$ & $1.1 \pm 0.6$ & 0.003 \\
\hline $\begin{array}{l}\text { C-peptide after } \\
\text { stimulation }(\mathrm{ng} / \mathrm{ml})\end{array}$ & $2.2 \pm 1.5$ & $3.6 \pm 1.7$ & $1.7 \pm 1.0$ & 0.000001 \\
\hline Hypothyroidism (n (\%)) & $3(3.7)$ & $0(0)$ & $3(5.4)$ & 0.33 \\
\hline $\begin{array}{l}\text { Subclinical } \\
\quad \text { hypothyroidism (n (\%)) }\end{array}$ & $9(11.1)$ & $1(4)$ & $8(14.4)$ & 0.37 \\
\hline Hyperthyroidism (n (\%)) & $3(3.7)$ & $0(0)$ & $3(5.4)$ & 0.33 \\
\hline TSH (mU/l) & $1.97(1.16-3.22)$ & $1.92(1.22-2.60)$ & $2.0(1.09-3.48)$ & 0.74 \\
\hline Women (mU/l) & $2.01(1.08-3.50)$ & $1.87(1.12-2.71)$ & $2.02(1.05-3.57)$ & 0.81 \\
\hline Men $(\mathrm{mU} / \mathrm{l})$ & $1.97(1.22-3.0)$ & $1.97(1.22-2.60)$ & $1.89(1.19-3.35)$ & 0.92 \\
\hline ATPO positive $(n(\%))$ & $30(37.5)$ & $4(16.6)$ & $26(46.4)$ & 0.01 \\
\hline Women $(n(\%))$ & $15(18.75)$ & $2(6.4)$ & $13(41.9)$ & 0.11 \\
\hline $\operatorname{Men}(n(\%))$ & $15(18.75)$ & $2(4.08)$ & $13(26.5)$ & 0.04 \\
\hline ATPO (IU/ml) & $0.38(0-35.9)$ & $0.03(0-0.6)$ & $0.74(0-280)$ & 0.04 \\
\hline Women (IU/ml) & $0.85(0-307.34)$ & $0.03(0-2.55)$ & $18.9(0-414.38)$ & 0.12 \\
\hline Men (IU/ml) & $0.28(0-15.79)$ & $0.02(0-0.50)$ & $0.45(0-29.81)$ & 0.15 \\
\hline
\end{tabular}

${ }^{*} P$ value in comparison with that of the group without autoantibodies. SBP, systolic blood pressure; DBP, diastolic blood pressure; TG, triglycerides; eGFR, glomerular filtration rate estimated using the Modification of Diet in Renal Disease (MDRD) study equation; ATPO; anti-thyroid peroxidase antibodies; TSH, thyroid-stimulating hormone.

with $\mathrm{O}$ blood group. Antibody titres were determined by serum dilution, and the titres are expressed as Juvenile Diabetes Foundation (JDF) units based on a reference sample of $80 \mathrm{JDF}$ obtained as a gift from the laboratory at Saint Vincent de Paul Hospital in Paris, France. Positivity was defined as greater than five JDF units. GADA and IA-2 were detected using commercial ELISA kits, GADA Assay (IgG) (positivity: >10 U/ml) and IA-2 Assay (IgG) (positivity: $>20 \mathrm{U} / \mathrm{ml}$ ) respectively (EUROIMMUN GmbH, Germany) (18).

ZnT8A were detected using the Luciferase Immunoprecipitation System (LIPS) method. The extracted DNA fragments containing two allelic variant forms of the ZnT8 antigen, aa 325 and aa 325-Arg-Trp, which are located at the C-terminus of the nZnT8 peptide molecule $(\mathrm{COOH}$-terminal portion of aa 268-369), were used. These fragments were cloned into a vector using a transcription set, Renilla Luciferase Assay System (E2820, Promega), resulting in a
ZnT8-antigen complex luciferase Renilla (Rluc-ZnT8). Biosynthesis of the antigen was then performed using a commercially available ZnT8 set of TNT SP6 Quick Coupled Transcription/Translation System (L2080, Promega). Later, sera were tested using a 96-well plate by properly applying the WHO standard dilution (250 WHO units/ml). The RlucZnT8 complex was added to all the wells, and the plate was incubated for $2 \mathrm{~h}$ at room temperature. Then, Protein A Sepharose was added to the wells, and the plate was incubated for $1 \mathrm{~h}$ at $+4{ }^{\circ} \mathrm{C}$ at 1000 r.p.m. After washing the wells with a wash buffer, lysis buffer was added. The plate was transferred to a luminometer, where the injectors with the added substrate (Coelenterazine) and luminescence were read at a wavelength of $480 \mathrm{~nm}$. The concentration of the antibodies was determined using the ZnT8 WHO standard curve, and the results are expressed in arbitrary units $(\mathrm{U} / \mathrm{ml})$ (WHO units/ml). This method was certified by 
the Diabetes Autoantibody Standardisation Program/Islet Autoantibodies Standardisation Program (DASP/IASP) with a specificity of $97.8 \%$ in adults.

Autoantibody analysis was carried out in the Immunopathology Laboratory at the Department of Paediatrics, Medical University of Lodz, Poland, which is a reference laboratory for the measurement of islet antibodies and is a regular participant in international proficiency testing programmes.

\section{TSH and thyroid peroxidase autoantibody assays}

The serum concentrations of TSH were determined using electrochemiluminescence ECLIA Elecsys analysers using MABs directed against the human TSH. The normal range was $0.27-4.2 \mathrm{mU} / \mathrm{l}$. In case of abnormal TSH concentrations, we evaluated free thyroid hormones (free triiodothyronine ( $\mathrm{fT}_{3}$ ) and free thyroxine $\left(\mathrm{fT}_{4}\right)$ ). Thyroid hormones were evaluated using electrochemiluminescence ECLIA Elecsys analysers. The range of normal values for adults was 3.1-6.8 pmol/1 for $\mathrm{fT}_{3}$ and $12-22 \mathrm{pmol} / \mathrm{l}$ for $\mathrm{fT}_{4}$. The evaluation of TSH and free thyroid hormones was done after adjusting the metabolic status of the patients (stable glycaemia and absence of ketones in the urine).

ATPO were detected using the ARCHITECT ATPO assay. A two-step immunoassay was used for the quantitative determination of ATPO in human serum using the Chemiluminescent Microparticle Immunoassay (CMIA) technology with flexible assay protocols, referred to as Chemiflex. In the first step, sample, assay diluent and TPOcoated paramagnetic microparticles were mixed and incubated. ATPO present in the sample binds to the TPO-coated microparticles. After washing, anti-human IgG acridiniumlabelled conjugate was added in the second step. Following another incubation and wash, pre-trigger and trigger solutions were added to the reaction mixture. The resulting chemiluminescent reaction was measured as relative light units (RLUs). A direct relationship exists between the amount of ATPO in the sample and the RLUs detected by the ARCHITECT immunoassay system optics. Results are expressed as international units per millilitre, and the upper level of normal range was $5.61 \mathrm{IU} / \mathrm{ml}$. Positive ATPO value was defined as ATPO level above $5.61 \mathrm{IU} / \mathrm{ml}$.

\section{Statistical analysis}

Statistical analysis was carried out using Statistical PL, version 8.0 (StatSoft, Inc., Tulsa, OK, USA). The results obtained for continuous variables are reported as means \pm s.D. for normally distributed data, as median values and
IQR for skewed data, or as the number and percentage of patients for categorical data. The Kolmogorov-Smirnov test with Lilliefors correction was used to test for normality. In analyses, a $P$ value $<0.05$ was considered to be statistically significant. In the statistical analysis, the following tests were used: Mann-Whitney $U$ test for comparison of non-parametric ordinal data, Student's $t$-test for comparison of normally distributed ordinal data, and $\chi^{2}$ test for comparison of categorical variables. Univariate regression analysis was used to assess the influence of selected variables on the occurrence of ATPO.

\section{Results}

In the study population, $70 \%(n=56)$ of the non-obese subjects with new-onset diabetes were positive for at least one of the four assessed markers of autoimmune diabetes (83.9\% GADA, 62.5\% ICA, 42.8\% IA-2A and 33.0\% ZnT8A). Additional analysis for the presence of ZnT8A revealed one more patient to be positive for autoantibodies.

In the study population, $37.5 \%(n=30)$ of the subjects were ATPO positive. The frequencies of clinical thyroid disease in ATPO-positive subjects were as follows: three had hypothyroidism and three had hyperthyroidism at diagnosis. Elevated concentrations of TSH, in the presence of normal concentrations of thyroid hormones and in the absence of clinical signs of thyroid disease (subclinical hypothyroidism), were found in nine of the ATPO-positive subjects (Table 1).

In the group with at least one islet autoantibody (LADA), we found higher HbA1c values $(11.4 \pm 2.4$ vs $9.9 \pm 2.5 \%, \quad P=0.01)$, lower total cholesterol levels $(5.0 \pm 1.0$ vs $5.8 \pm 1.7 \mathrm{mmol} / \mathrm{l}, P=0.03)$, and lower serum fasting $(1.1 \pm 0.6$ vs $1.7 \pm 0.9 \mathrm{ng} / \mathrm{ml}, P=0.003)$ and stimulated $(1.7 \pm 1.0$ vs $3.6 \pm 1.7 \mathrm{ng} / \mathrm{ml}, P=0.000001)$ C-peptide levels in comparison with those in the group without islet autoantibodies. There were more ATPO-positive subjects (46.4 vs $16.6 \%, P=0.01$ ) in the LADA group and among men ( 39.4 vs $12.5 \%, P=0.04)$ with autoimmune diabetes. In the LADA group, $25 \%(n=14)$ of the subjects had overt or subclinical thyroid disease (Table 1).

Comparison of LADA patients according to the occurrence of ZnT8A revealed significantly lower stimulated C-peptide levels $(1.30 \pm 0.72$ vs $1.98 \pm 1.20 \mathrm{ng} / \mathrm{ml}$, $P=0.02$ ), higher titres of ATPO (172.7 (IQR: 0.36-410.4) vs 92.4 (IQR: $0-23.7) \mathrm{IU} / \mathrm{ml}, P=0.001$ ), and more frequent occurrence (89.4 vs $51.4 \%, P=0.005$ ) and higher titres of ICA (80 (IQR: 40-160) vs 20 (IQR: 0-40) IU/ml, $P=0.00006$ ) in the ZnT8A-positive group (Table 2). 
Table 2 Comparison between the ZnT8A-positive group and the ZnT8A-negative group among LADA patients (median and IQR or mean \pm s.D. or percentage of patients).

\begin{tabular}{|c|}
\hline$n$ \\
\hline Sex (women/men) \\
\hline Age (years) \\
\hline BMI $\left(\mathrm{kg} / \mathrm{m}^{2}\right)$ \\
\hline Waist (cm) \\
\hline Women $(\mathrm{cm})$ \\
\hline Men $(\mathrm{cm})$ \\
\hline SBP $(\mathrm{mmHg})$ \\
\hline $\mathrm{DBP}(\mathrm{mmHg})$ \\
\hline $\mathrm{HbA1c}(\%)$ \\
\hline Cholesterol (mmol/l) \\
\hline TG $(\mathrm{mmol} / \mathrm{l})$ \\
\hline LDL (mmol/l) \\
\hline $\mathrm{HDL}(\mathrm{mmol} / \mathrm{l})$ \\
\hline Creatinine (mg/dl) \\
\hline GFR (MDRD) (ml/min) \\
\hline Fasting C-peptide (ng/ml) \\
\hline $\begin{array}{l}\text { C-peptide after stimulation } \\
(\mathrm{ng} / \mathrm{ml})\end{array}$ \\
\hline Positive GADA ( $n(\%))$ \\
\hline Positive ICA ( $n(\%))$ \\
\hline Positive IA-2A ( $(\%))$ \\
\hline GADA (U/ml) \\
\hline ICA (JDF) \\
\hline $\mathrm{IA}-2 \mathrm{~A}(\mathrm{U} / \mathrm{ml})$ \\
\hline $\mathrm{TSH}(\mathrm{mU} / \mathrm{l})$ \\
\hline ATPO positive $(n(\%))$ \\
\hline ATPO $(\mathrm{mU} / \mathrm{l})$ \\
\hline
\end{tabular}

\begin{tabular}{c}
\hline ZnT8A-positive group \\
\hline 19 \\
$9 / 10$ \\
$43(37-51)$ \\
$23.2(20.0-26.0)$ \\
$78.8 \pm 10.3$ \\
$89.4 \pm 5.1$ \\
$110(110-120)$ \\
$70(70-80)$ \\
$11.6 \pm 2.2$ \\
$5.3 \pm 0.8$ \\
$1.1(0.9-1.5)$ \\
$3.5 \pm 1.2$ \\
$1.3 \pm 0.4$ \\
$0.78 \pm 0.14$ \\
$101.3 \pm 21.1$ \\
$0.92 \pm 0.45$ \\
$1.30 \pm 0.72$ \\
$16(84.2)$ \\
$17(89.4)$ \\
$9(47.3)$ \\
$522.3(29.3-714.0)$ \\
$80(40-160)$ \\
$19.1(10.0-745.1)$ \\
$1.98(1.09-4.21)$ \\
$12(63.1)$ \\
$172.7(0.36-410.4)$ \\
\end{tabular}

\begin{tabular}{c}
\hline ZnT8-negative group \\
\hline 37 \\
$14 / 23$ \\
$45(37-44)$ \\
$24.0(23.0-26.0)$ \\
$79.3 \pm 8.5$ \\
$91.6 \pm 9.9$ \\
$112(110-120)$ \\
$75(70-80)$ \\
$11.3 \pm 2.5$ \\
$4.9 \pm 1.0$ \\
$1.3(0.8-1.6)$ \\
$3.2 \pm 0.8$ \\
$1.3 \pm 0.5$ \\
$0.81 \pm 0.15$ \\
$102.3 \pm 26.9$ \\
$1.29 \pm 0.74$ \\
$1.98 \pm 1.20$ \\
$31(83.8)$ \\
$18(51.4)$ \\
$15(41.6)$ \\
$282.8(27.5-533.5)$ \\
$20(0-40)$ \\
$17.3(7.7-42.6)$ \\
$2.70(1.05-3.43)$ \\
$14(37.8)$ \\
$92.4(0-23.7)$ \\
\end{tabular}

\begin{tabular}{ll}
\hline P value \\
\hline- \\
0.49 \\
0.55 \\
0.48 \\
\\
0.88 \\
0.54 \\
0.87 \\
0.18 \\
0.73 \\
0.43 \\
0.74 \\
0.17 \\
0.89 \\
0.51 \\
0.88 \\
0.05 \\
0.02 \\
0.96 \\
0.005 \\
0.68 \\
0.12 \\
0.00044 \\
0.24 \\
0.74 \\
0.07 \\
0.02 \\
\hline
\end{tabular}

SBP, systolic blood pressure; DBP, diastolic blood pressure; TG, triglycerides; eGFR, glomerular filtration rate estimated using the Modification of Diet in Renal Disease (MDRD) study equation; GADA, glutamic acid decarboxylase autoantibodies; ICA, autoantibodies to islet cells; IA-2A, antibodies to tyrosine phosphatase; ZnT8A, zinc transporter type 8 autoantibodies; ATPO, anti-thyroid peroxidase antibodies; TSH, thyroid-stimulating hormone.

We assessed the relationship between the presence of ATPO and that of ICA using a univariate regression analysis model. The presence of $\mathrm{ZnT8A}(\mathrm{OR}=5.48,95 \%$ CI: $1.65-18.14, P=0.006)$ and GADA $(\mathrm{OR}=3.42,95 \% \mathrm{CI}$ : 1.09-10.71, $P=0.03$ ) was found to be related to positive titres of ATPO (Table 3).

\section{Discussion}

In the present study, we demonstrated the prevalence of diabetes with positive autoimmune markers to be $70 \%$ in non-obese newly diagnosed diabetic patients aged $>35$ years when assessing four autoantibodies (GADA, ICA, IA-2A and ZnT8A). Similar to other publications, our results demonstrated that in adult-onset diabetic patients GADA are the most common autoantibodies $(19,20)$. ZnT8A have been found to be present in a significant percentage of patients. Additional testing for the presence of ZnT8A identified only one subject predetermined to be antibody negative when evaluating GADA, ICA and IA-2A.
In their study, Andersson et al. (13) discovered that in childhood diabetes, ZnT8A were present in $65 \%$ of the subjects. Similarly to our study population, only $3.4 \%$ of children were found to be positive for ZnT8A as the only islet antibody. By contrast, in Argentinian LADA patients, Trabucchi et al. (21) demonstrated that $10.7 \%$ had ZnT8A as the only humoral marker. Through screening, Lampasona et al. (9) demonstrated that ZnT8A coexisted

Table 3 Relationship between the presence of ATPO and that of islet cell antibodies: univariate regression analysis model.

\begin{tabular}{lllll}
\hline & \multicolumn{1}{c}{ OR (95\%CI) } & & $\boldsymbol{P}$ \\
\cline { 1 - 2 } GADA & & $3.42(1.09-10.71)$ & & 0.03 \\
ICA & $2.46(0.92-6.55)$ & & 0.06 \\
IA-2A & $1.10(0.41-2.96)$ & & 0.83 \\
ZnT8A & $\mathbf{5 . 4 8 ( 1 . 6 5 - 1 8 . 1 4 )}$ & $\mathbf{0 . 0 0 6}$ \\
\hline
\end{tabular}

GADA, glutamic acid decarboxylase autoantibodies; ICA, autoantibodies to islet cells; IA-2A, antibodies to tyrosine phosphatase; ZnT8A, zinc transporter type 8 autoantibodies; ATPO, anti-thyroid peroxidase antibodies. 
with GADA and IA-2A in $18.6 \%$ of the LADA patients. Moreover, these were found in younger patients, and additional detection for the presence of ZnT8A as a third autoantibody predicted more severe $\beta$-cell dysfunction. In the present study, we demonstrated that ZnT8A coexisted more frequently with ICA. Patients positive for ZnT8A also had lower stimulated C-peptide levels in comparison with other LADA patients.

AIT is common in T1DM (15-30\%), as both are organspecific autoimmune diseases $(22,23,24)$. Their aetiology may involve common genetic factors $(17,25)$. The probability of thyroiditis increases with age; therefore, the occurrence of positive ATPO in LADA is more common than in younger T1DM patients (26). In the present study, in the LADA group, we found a significant proportion of patients (46.4\%) to be ATPO positive during the diagnosis of diabetes and $25 \%$ of these patients to have overt or subclinical thyroid disease. Jin et al. (17) demonstrated the incidence of thyroid antibody positivity in $21.5 \%$ of LADA patients, with $11.1 \%$ of them presenting thyroid dysfunction. The dynamics of humoral autoimmunity to islet autoantigens in association with anti-thyroid autoimmunity in T1DM is not clear. More frequent occurrence of positive anti-thyroid antibodies has been reported in T1DM patients as well as in LADA subjects with higher GADA titres than in those without GADA or with lower titres $(15,17,27)$. Kawasaki et al. (27) also found that persistent positivity for GADA could be associated with an increased risk of developing anti-thyroid autoimmunity in the future. The association between GADA and thyroid antibodies may be explained by the fact that the glutamic acid decarboxylase antigen is found in the thyroid gland and could be expressed on thyroid epithelial cells to helper T-lymphocyte to produce GADA (28). In the present study, we confirmed the relationship between the occurrence of GADA and that of ATPO in newly diagnosed autoimmune diabetic patients aged $>35$ years.

Interestingly, we demonstrated the strongest relationship between the presence of positive ZnT8A and that of ATPO. Furthermore, we demonstrated that ATPO titres were statistically higher in LADA patients positive for ZnT8A than in the group without ZnT8A. To our knowledge, this is the first study to prove that a strong relationship exists between the presence of these two autoantibodies (ZnT8A and ATPO) in newly diagnosed diabetic adult patients. Independently, in a large population of children with new onset of T1DM, Jonsdottir et al. (29) discovered that both types of thyroid autoantibodies (ATPO and anti-thyroglobulin antibody ATg) were associated with GADA and ZnT8A. Zinc modulates the expression of many genes within the immune cells and is an essential element for immune function. Zinc transporters control its homoeostasis. ZnT8 is localised on insulin-containing secretory granule membranes (30). We still do not know whether the same process could be responsible for the development of thyroiditis and share polymorphisms in the SLC30A8 gene. This seems probable, as the expression of ZnT8 has also been demonstrated in the cubical epithelium of thyroid follicles and in the cortex of adrenal glands (31). This finding suggests its widespread role in the endocrine system. An interesting question to be unravelled is the ability to distinguish the islet cell autoantibodies in a healthy population with the aim of identifying subjects prone to developing autoimmune thyroid disease. A longitudinal study is also necessary to determine whether the presence of GADA and ZnT8A in patients with Hashimoto's thyroiditis can predict the onset of diabetes.

The authors of previous studies have indicated the relevance of regular screening in LADA population, especially with high GADA titres, to detect patients with asymptomatic thyroid dysfunction $(15,17)$. Our results have shown that the presence of not only GADA but also ZnT8A predicts the possibility of AITD occurring in LADA patients. We conclude that the presence of ZnT8A should sensitise physicians to test for the presence of autoimmune thyroid disease. We proved that the presence of ZnT8A does not significantly increase the sensitivity of autoimmune diabetes diagnosis in adults, but that it increases the risk of concomitant thyroiditis.

The present study not only has novel aspects, but also has a few limitations. First, the study was carried out in a substantially small group of subjects. Furthermore, only non-obese patients aged $>35$ years were recruited. Some LADA criteria specify the adult age to be more than 35 years $(5,32)$. However, no difference in autoimmune status and clinical perspectives could be detected in the study population in comparison with younger subjects. LADA patients are mostly lean, but obesity does not exclude autoimmunity (10). We performed thyroid screening using TSH and ATPO assays as commonly recommended. We did not evaluate ATg. Kucera et al. (26) indicated that there is a significant difference between LADA and T2DM patients with regard to ATPO but not to ATg. We did not detect for the presence of other organ-specific autoantibodies characteristic of autoimmune polyglandular syndrome. Zampetti et al. (15) found steroid 21-hydroxylase in only $3.4 \%$ of the LADA patients with high GADA titres and not in subjects with low GADA titres. However, it would be interesting to evaluate other markers of autoimmunity and correlate them with ZnT8A. 


\section{Conclusions}

The occurrence of positive thyroid peroxidase antibodies is closely associated with LADA. The presence of GADA and especially of ZnT8A predisposes non-obese adultonset diabetic patients aged $>35$ years to AITD.

\section{Declaration of interest}

The authors declare that there is no conflict of interest that could be perceived as prejudicing the impartiality of the research reported.

\section{Funding}

This study was supported by a grant (number 502-14-02234382-09) from Poznan University of Medical Sciences, Poland.

\section{Acknowledgements}

The authors thank the staff at Immunopathology Laboratory at the Department of Paediatrics, Medical University of Lodz, Poland, for carrying out the antibody assays.

\section{References}

1 Alberti KG \& Zimmet PZ. Definition, diagnosis and classification of diabetes mellitus and its complications. Part 1: diagnosis and classification of diabetes mellitus provisional report of a WHO consultation. Diabetic Medicine 199815 539-553. (doi:10.1002/ (SICI) 1096-9136(199807)15:7 < 539::AID-DIA668>3.0.CO;2-S)

2 Naik RG \& Palmer JP. Latent autoimmune diabetes in adults (LADA). Reviews in Endocrine \& Metabolic Disorders 20034 233-241. (doi:10.1023/ A:1025148211587)

3 Zimmet PZ. The pathogenesis and prevention of diabetes in adults. Genes, autoimmunity, and demography. Diabetes Care 199518 1050-1064.

4 Pozzilli P \& Di Mario U. Autoimmune diabetes not requiring insulin at diagnosis (latent autoimmune diabetes of the adult): definition, characterization, and potential prevention. Diabetes Care 200124 1460-1467. (doi:10.2337/diacare.24.8.1460)

5 Tuomi T, Carlsson A, Li H, Isomaa B, Miettinen A, Nilsson A, Nissen M, Ehrnstrom BO, Forsen B, Snickars B et al. Clinical and genetic characteristics of type 2 diabetes with and without GAD antibodies. Diabetes 199948 150-157. (doi:10.2337/diabetes.48.1.150)

6 Maruyama T, Shimada A, Kanatsuka A, Kasuga A, Takei I, Yokoyama J \& Kobayashi T. Multicenter prevention trial of slowly progressive type 1 diabetes with small dose of insulin (the Tokyo study): preliminary report. Annals of the New York Academy of Sciences 20031005 362-369. (doi:10.1196/annals.1288.060)

7 Chaillous L, Bouhanick B, Kerlan V, Mathieu E, Lecomte P, Ducluzeau PH, Delamaire M, Sonnet E, Maugendre D, Marechaud R et al. Clinical and metabolic characteristics of patients with latent autoimmune diabetes in adults (LADA): absence of rapid $\beta$-cell loss in patients with tight metabolic control. Diabetes \& Metabolism 201036 64-70. (doi:10.1016/j.diabet.2009.07.004)

8 Fourlanos S, Dotta F, Greenbaum CJ, Palmer JP, Rolandsson O, Colman PG \& Harrison LC. Latent autoimmune diabetes in adults (LADA) should be less latent. Diabetologia 200548 2206-2212. (doi:10.1007/s00125-005-1960-7)

9 Lampasona V, Petrone A, Tiberti C, Capizzi M, Spoletini M, di Pietro S, Songini M, Bonicchio S, Giorgino F, Bonifacio E et al. Zinc transporter 8 antibodies complement GAD and IA-2 antibodies in the identification and characterization of adult-onset autoimmune diabetes: non insulin requiring autoimmune diabetes (NIRAD) 4. Diabetes Care 201033 104-108. (doi:10.2337/dc08-2305)

10 Juneja R, Hirsch IB, Naik RG, Brooks-Worrell BM, Greenbaum CJ \& Palmer JP. Islet cell antibodies and glutamic acid decarboxylase antibodies, but not the clinical phenotype, help to identify type $1(1 / 2)$ diabetes in patients presenting with type 2 diabetes. Metabolism 2001 50 1008-1013. (doi:10.1053/meta.2001.25654)

11 Borg H, Gottsater A, Fernlund P \& Sundkvist G. A 12-year prospective study of the relationship between islet antibodies and $\beta$-cell function at and after the diagnosis in patients with adult-onset diabetes. Diabetes 200251 1754-1762. (doi:10.2337/diabetes.51.6.1754)

12 Chimienti F, Favier A \& Seve M. ZnT-8, a pancreatic $\beta$-cell-specific zinc transporter. Biometals 200518 313-317. (doi:10.1007/s10534005-3687-9)

13 Andersson C, Vaziri-Sani F, Delli A, Lindblad B, Carlsson A, Forsander G, Ludvigsson J, Marcus C, Samuelsson U, Ivarsson S et al. Triple specificity of ZnT8 autoantibodies in relation to HLA and other islet autoantibodies in childhood and adolescent type 1 diabetes. Pediatric Diabetes 201314 97-105. (doi:10.1111/j.1399-5448.2012. 00916.x)

14 Rosario PW, Reis JS, Borges MA, Amim R, Calsolari MR, Silva SC \& Purisch S. Extrapancreatic autoimmunity in patients with latent autoimmune diabetes of adults. Diabetes Care 200528 496-497. (doi:10.2337/diacare.28.2.496-a)

15 Zampetti S, Capizzi M, Spoletini M, Campagna G, Leto G, Cipolloni L, Tiberti C, Bosi E, Falorni A \& Buzzetti R. GADA titer-related risk for organ-specific autoimmunity in LADA subjects subdivided according to gender (NIRAD study 6). Journal of Clinical Endocrinology and Metabolism 201297 3759-3765. (doi:10.1210/jc.2012-2037)

16 Maioli M, Pes GM, Delitala G, Puddu L, Falorni A, Tolu F, Lampis R, Orru V, Secchi G, Cicalo AM et al. Number of autoantibodies and HLA genotype, more than high titers of glutamic acid decarboxylase autoantibodies, predict insulin dependence in latent autoimmune diabetes of adults. European Journal of Endocrinology 2010163 541-549. (doi:10.1530/EJE-10-0427)

17 Jin P, Huang G, Lin J, Yang L, Xiang B, Zhou W \& Zhou Z. High titre of antiglutamic acid decarboxylase autoantibody is a strong predictor of the development of thyroid autoimmunity in patients with type 1 diabetes and latent autoimmune diabetes in adults. Clinical Endocrinology 201174 587-592. (doi:10.1111/j.1365-2265.2011.03976.x)

18 Rogowicz-Frontczak A, Zozulinska-Ziolkiewicz D, Niedzwiecki P, Litwinowicz M \& Wierusz-Wysocka B. Does glucagon stimulation test help to predict autoimmunity in newly diagnosed non obese adults with diabetes? Experimental and Clinical Endocrinology \& Diabetes 2012 120 428-434. (doi:10.1055/s-0032-1308979)

19 Davis TM, Wright AD, Mehta ZM, Cull CA, Stratton IM, Bottazzo GF, Bosi E, Mackay IR \& Holman RR. Islet autoantibodies in clinically diagnosed type 2 diabetes: prevalence and relationship with metabolic control (UKPDS 70). Diabetologia 200548 695-702. (doi:10.1007/ s00125-005-1690-x)

20 Bottazzo GF, Bosi E, Cull CA, Bonifacio E, Locatelli M, Zimmet P, Mackay IR \& Holman RR. IA-2 antibody prevalence and risk assessment of early insulin requirement in subjects presenting with type 2 diabetes (UKPDS 71). Diabetologia 200548 703-708. (doi:10.1007/ s00125-005-1691-9)

21 Trabucchi A, Faccinetti NI, Guerra LL, Puchulu FM, Frechtel GD Poskus E \& Valdez SN. Detection and characterization of ZnT8 autoantibodies could help to screen latent autoimmune diabetes in adult-onset patients with type 2 phenotype. Autoimmunity 201245 137-142. (doi:10.3109/08916934.2011.604658)

22 Chang CC, Huang CN \& Chuang LM. Autoantibodies to thyroid peroxidase in patients with type 1 diabetes in Taiwan. European Journal of Endocrinology 1998139 44-48. (doi:10.1530/eje.0.1390044) 
23 Barker JM, Yu J, Yu L, Wang J, Miao D, Bao F, Hoffenberg E, Nelson JC, Gottlieb PA, Rewers M et al. Autoantibody "subspecificity" in type 1 diabetes: risk for organ-specific autoimmunity clusters in distinct groups. Diabetes Care 200528 850-855. (doi:10.2337/diacare.28.4.850)

24 Kordonouri O, Klinghammer A, Lang EB, Gruters-Kieslich A, Grabert M $\&$ Holl RW. Thyroid autoimmunity in children and adolescents with type 1 diabetes: a multicenter survey. Diabetes Care 200225 1346-1350. (doi:10.2337/diacare.25.8.1346)

25 Huang W, Connor E, Rosa TD, Muir A, Schatz D, Silverstein J, Crockett S, She JX \& Maclaren NK. Although DR3-DQB1*0201 may be associated with multiple component diseases of the autoimmune polyglandular syndromes, the human leukocyte antigen DR4DQB ${ }^{*} 0302$ haplotype is implicated only in $\beta$-cell autoimmunity. Journal of Clinical Endocrinology and Metabolism 199681 2559-2563.

26 Kucera P, Novakova D, Behanova M, Novak J, Tlaskalova-Hogenova $\mathrm{H}$ \& Andel M. Gliadin, endomysial and thyroid antibodies in patients with latent autoimmune diabetes of adults (LADA). Clinical and Experimental Immunology 2003133 139-143. (doi:10.1046/j.1365-2249. 2003.02205.x)

27 Kawasaki E, Yasui J, Tsurumaru M, Takashima H, Ikeoka T, Mori F, Akazawa S, Ueki I, Kobayashi M, Kuwahara H et al. Sequential elevation of autoantibodies to thyroglobulin and glutamic acid decarboxylase in type 1 diabetes. World Journal of Diabetes 20134 227-230.

28 Kawasaki E, Takino H, Yano M, Uotani S, Matsumoto K, Takao Y, Yamaguchi Y, Akazawa S \& Nagataki S. Autoantibodies to glutamic acid decarboxylase in patients with IDDM and autoimmune thyroid disease. Diabetes 199443 80-86. (doi:10.2337/diab.43.1.80)

29 Jonsdottir B, Andersson C, Carlsson A, Delli A, Forsander G, Ludvigsson J, Marcus C, Samuelsson U, Ortqvist E, Lernmark A et al. Thyroid autoimmunity in relation to islet autoantibodies and HLA-DQ genotype in newly diagnosed type 1 diabetes in children and adolescents. Diabetologia 201356 1735-1742. (doi:10.1007/s00125013-2934-9)

30 Kawasaki E. ZnT8 and type 1 diabetes. Endocrine Journal 201259 531-537. (doi:10.1507/endocrj.EJ12-0069)

31 Murgia C, Devirgiliis C, Mancini E, Donadel G, Zalewski P \& Perozzi G. Diabetes-linked zinc transporter ZnT8 is a homodimeric protein expressed by distinct rodent endocrine cell types in the pancreas and other glands. Nutrition, Metabolism, and Cardiovascular Diseases 200919 431-439. (doi:10.1016/j.numecd.2008.09.004)

32 Gale EA. Latent autoimmune diabetes in adults: a guide for the perplexed. Diabetologia 200548 2195-2199. (doi:10.1007/ s00125-005-1954-5)

Received 3 November 2013

Revised version received 24 January 2014

Accepted 30 January 2014 